
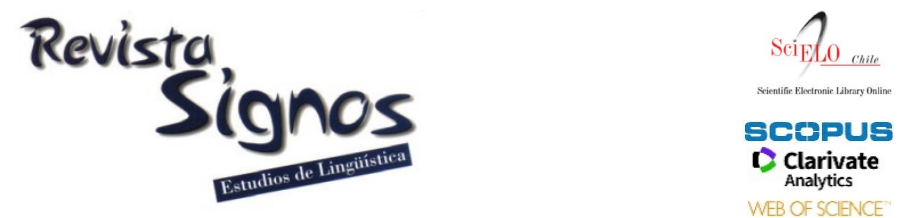

\title{
Estructura hipertextual y habilidad lectora: Análisis de la producción digital de textos argumentativos en estudiantes de $4^{\circ}$ Año Medio
}

\author{
Hypertext structure and reading skills: Analysis of 12 th \\ graders' argumentative texts digital production
}

Gabriela Vallejos

Carrasco

UNIVERSIDAD DE CONCEPCIÓN

CHILE

gvallejos@udec.cl

\author{
Mónica Véliz \\ de Vos \\ UNIVERSIDAD DE CONCEPCIÓN \\ CHILE \\ mveliz@udec.cl
}

\author{
Katia Sáez \\ Carrillo \\ UNIVERSIDAD DE CONCEPCIÓN \\ CHILE \\ ksaez@udec.cl
}

Recibido: 05-XII-2018 / Aceptado: 07-VI-2020

DOI: $10.4067 /$ S0718-09342021000100097

\section{Resumen}

El estudio examina la producción textual digital de ensayos argumentativos en el marco de una tarea del tipo 'leer para escribir'. Se propone relacionar la estructura del hipertexto que utilizan los sujetos para estimular la escritura y su nivel de habilidad lectora con los índices del proceso de producción textual y la calidad de los ensayos producidos. Se solicitó a 68 estudiantes de $4^{\circ}$ Año Medio de un liceo de Concepción que redactaran un ensayo argumentativo dialéctico a partir de la lectura de un reportaje en dos formatos de diferente estructura hipertextual: jerarquizada y en red. El proceso de producción fue registrado mediante un keylogger. Se consideró la cantidad y duración de pausas, la cantidad de revisiones y la cantidad y duración de las visitas al hipertexto. Los productos escritos fueron evaluados por un juez experto que aplicó una pauta de evaluación dividida en tres niveles textuales (microestructural, macroestructural y superestructural). Los resultados obtenidos sugieren que la estructura del recurso de lectura hipertextual no se relaciona de forma significativa con los procesos ni con los productos escritos. La habilidad lectora, en tanto, correlaciona significativamente con la calidad de los productos escritos, aunque dicha correlación es baja. Para futuras investigaciones con el método de keystroke logging, se recomienda realizar análisis más finos, focalizados en uno o dos índices y registrar patrones de ocurrencia, localización y fluidez.

Palabras Clave: Producción escrita, leer para escribir, hipertexto, keystroke logging. 


\begin{abstract}
The aim of this study is to examine the digital textual production of argumentative essays in the context of a 'reading to write' task type. Our main goal is to relate the structure of the hypertext used by individuals to stimulate writing and the level of reading ability with the process indices of textual production and the quality of essays made. For this purpose, we asked sixty-eight 12th graders from a high school in Concepcion to write a dialectical argumentative essay after reading a two-style report with different hypertextual structure -hierarchical and network. The writing production process was captured through a keystroke logging. The number and duration of pauses and hypertext's views and number of revisions were registered and analyzed. Meanwhile, the writing production was evaluated by an expert who used an assessment instrument divided into three textual levels (microstructural, macrostructural and superstructural cognitive-discursive). The results showed that hypertext structure is not significantly related neither in writing process measurements nor final essays quality. Nevertheless, reading skill level significantly correlates with essays' quality, although correlation is low. It is recommended for future research in keystroke logging more focused analysis on few measurements and register occurrence patterns, location and fluency.
\end{abstract}

Key Words: Written production, reading to write, hyertext, keystroke logging.

\title{
INTRODUCCIÓN
}

Los enfoques acerca de la actividad de escritura han evolucionado en relación a épocas anteriores. Inicialmente, el acento estaba en el producto con foco en la superficie textual (ortografía, puntuación y gramática) (Parodi, 2003). Posteriormente, éste se desplazó hacia los procesos cognitivos implicados en la tarea de escritura, a los que se accedía mediante protocolos de pensamiento en voz alta (Flower \& Hayes, 1981). Actualmente, se piensa que la actividad de escribir involucra componentes antes no considerados, por ejemplo, la memoria operativa del escritor (Alamargot, Caporossi, Chesnet \& Ros, 2011), los materiales y el medio de transcripción dentro del entorno de la tarea de escritura y la motivación del escritor (Hayes, 1996, 2012).

Además, se debe considerar que en el ambiente escolar, el uso de Internet se ha vuelto masivo y su acceso cada vez más inmediato a través de dispositivos de uso cotidiano (Cassany \& Ayala, 2008). Para producir los textos que les exigen las instituciones educativas, los estudiantes leen hipertextos, es decir:

"un texto compuesto de bloques de palabras (o de imágenes) electrónicamente unidos mediante múltiples trayectos, cadenas o recorridos en una textualidad abierta, eternamente inacabada y descrita con términos como enlace, nodo, red, trama y trayecto" (Landow, 2009: 24).

En línea con lo planteado por O’Hara, Taylor, Newman y Sellen (2002) y Hayes (2012), se plantea que las características de los recursos de lectura utilizados influyen de alguna manera en la actividad de escritura. Burin, Kahan, Irrazabal y Saux (2010) 
apoyan esta visión con su hallazgo de que la estructura hipertextual leída influye en los resultados en comprensión.

La propuesta de este estudio amplía el alcance de las investigaciones antes mencionadas, considerando la tarea dada a los sujetos, quienes no solo deben comprender un hipertexto con una organización determinada, sino también ser capaces de tomar una posición frente a lo leído y comunicarla al lector mediante la producción de un ensayo. En esta línea, Parodi (2007) investigó las relaciones entre la comprensión y la producción de textos argumentativos en escolares de $8^{\circ}$ Año Básico de Valparaíso desde una perspectiva cognitiva y discursiva. Uno de los objetivos de su investigación fue buscar correlaciones entre los procesos de lectura y escritura en sus diferentes niveles (microestructural, macroestructural y superestructural). Los resultados mostraron coeficientes significativos entre lectura y escritura en todos los niveles analizados. En relación con las habilidades demostradas por los estudiantes, los puntajes más altos fueron logrados en el nivel de la coherencia local, mientras que la estructura organizacional de la argumentación (tesis, argumentos, conclusión) fue el de mayor dificultad para los estudiantes, tanto en lectura como en escritura.

Nuestro estudio busca obtener información acerca del proceso de producción que llevan a cabo sujetos de diferente nivel de habilidad lectora en una tarea híbrida que involucra alternar la escritura de un texto argumentativo con la lectura crítica de un recurso hipertextual con diferente estructura. El objetivo es determinar si la estructura del hipertexto y la habilidad lectora influyen en los índices del proceso de producción textual y en la calidad general de los ensayos escritos por un grupo de estudiantes de cuarto año medio de la ciudad de Concepción ${ }^{1}$. Se recogen los hallazgos de Burin et al. (2010) y de Parodi (2007) para sostener la hipótesis de que tanto la estructura del hipertexto como la habilidad lectora influirán en los índices del proceso de escritura y en la calidad general de los productos escritos.

A continuación se realizará una revisión de las principales líneas teóricas en las que se fundamenta la investigación, se describirá la metodología utilizada, y se darán a conocer los resultados obtenidos. Se finaliza con la discusión de dichos resultados y las conclusiones.

\section{Marco teórico}

\subsection{Constructo leer para escribir}

Las tareas de escritura sin una actividad de lectura asociada son difícilmente concebibles en entornos naturales, tales como los educativos y profesionales. En el enfoque 'leer para escribir' (Flower, 1987) se estudian las actividades híbridas en que los sujetos deben leer textos para crear uno nuevo que integre ideas de los textos leídos con sus propias ideas (Stein, 1989). 
De acuerdo a Delaney (2008), desde la perspectiva constructivista, la lectura y la escritura se conciben como procesos de construcción de significado. El lector/escritor establece nuevos significados a partir de las lecturas, los que luego articulará sobre el papel. Según esta aproximación, el solo hecho de poseer las habilidades de lectura y escritura de forma independiente no es garantía suficiente para desempeñar con éxito las tareas de leer para escribir.

\subsection{Modelo de Hayes (2012)}

El modelo de Hayes (2012), última reformulación del modelo original presentado por el autor en 1981 junto a Linda Flower, es el fundamento teórico base de nuestro estudio. El nuevo modelo incorpora la memoria de trabajo, la motivación y el proceso de transcripción; mientras que los procesos de monitoreo, planificación y revisión fueron removidos.

Tres niveles se integran en el modelo:

1) El nivel de control, compuesto por procesos que determinan a otros procesos, tales como la motivación; el establecimiento de las metas de escritura; el plan actual, que es el conjunto de metas para crear el texto en ejecución que está almacenado en la memoria, y los esquemas de escritura.

Para Hayes, resulta obvio que la motivación es necesaria para tener la buena voluntd de involucrarse en una tarea de escritura. El que la gente escriba o no, la extensión de los que escriben y cuánto se preocupan de la calidad de su escrito dependen de su motivación. En su nuevo modelo, establece claramente que la motivación afecta al establecimiento de metas de planificación, escritura y revisión.

Los esquemas de tareas de escritura, tales como revisar, tomar notas, resumir, etc., probablemente se encuentran almacenados en la memoria a largo plazo, donde se van enriqueciendo producto de nuevas experiencias y aprendizajes. Aquí se incluyen también las metas de las tareas de lectura necesarias para llevar a cabo la escritura.

2) El nivel de proceso, que se divide en dos subniveles: el proceso de escritura, que integra los procesos mentales internos utilizados en la tarea de escritura, y el entorno de la tarea, que incluye los contextos físicos, culturales y sociales de los procesos de escritura.

El proceso de escritura integra al proponente, un dispositivo que formula ideas no verbales; el traductor, que representa las ideas en lenguaje; el transcriptor, que las representa como signos lingüísticos gráficos; y el evaluador, que examina los productos de los tres procesos anteriores.

El entorno de la tarea, en tanto, está compuesto de colaboradores y críticos, además de los recursos materiales tales como libros, artículos, páginas web, apuntes, 
esquemas, entre otros materiales recolectados o preparados por el escritor, todos los cuales proporcionan sugerencias para el texto en ejecución ante el proponente. En este nivel, la cultura en que está inmerso el escritor es muy importante en la determinación de los procedimientos que lleva a cabo para escribir y a quién acude en caso de necesitar ayuda. El texto escrito hasta el momento es una fuente de consulta a medida que se avanza en el proceso. La transcripción también juega un papel importante en cómo se llevará a cabo el proceso, dependiendo del grado de dominio que tenga el escritor del método de transcripción escogido.

3) El nivel de recursos, que abarca los medios necesarios para realizar la escritura y otras tareas distintas como la memoria de largo plazo, la memoria de trabajo y la atención.

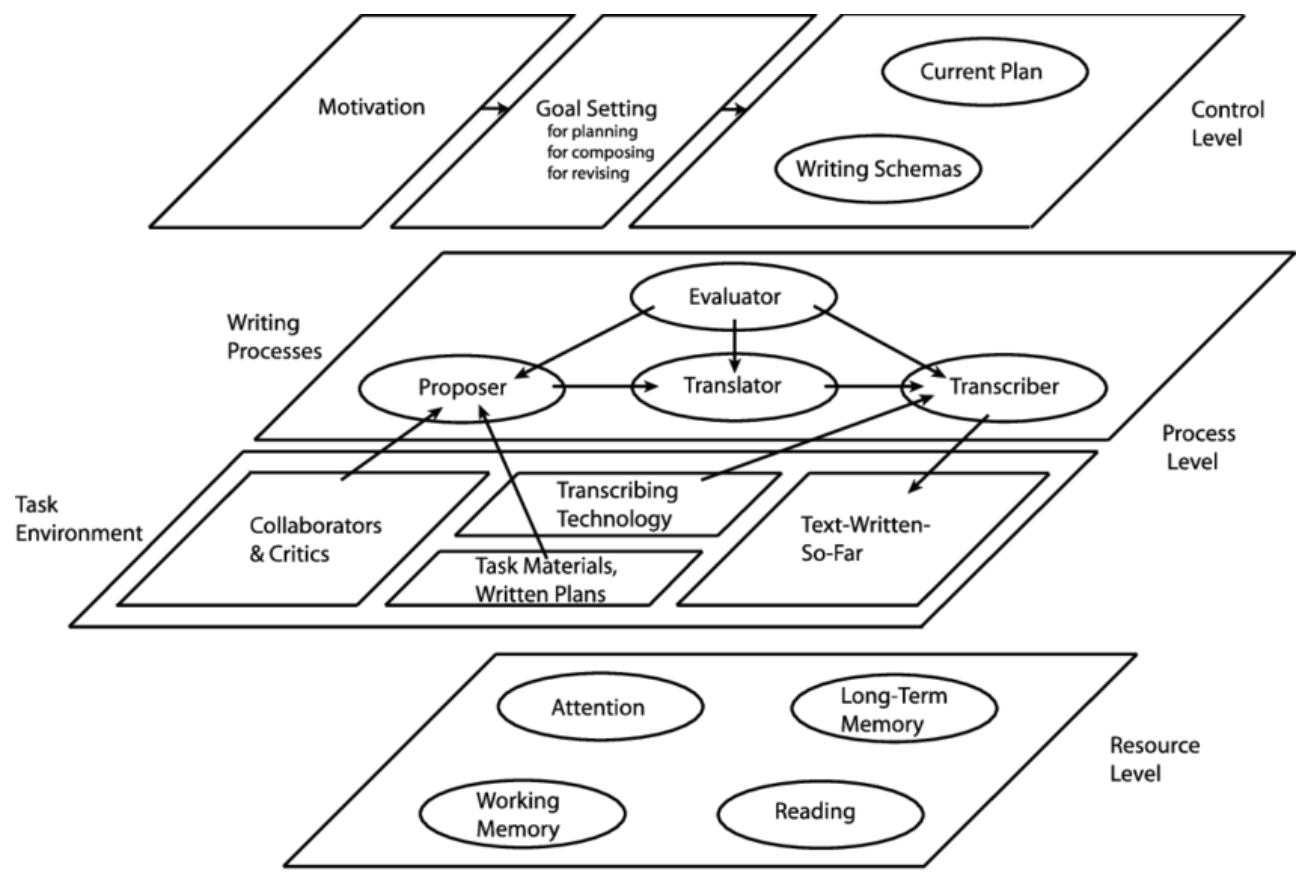

Figura 1. Modelo de escritura de Hayes (2012: 371).

\subsection{El texto argumentativo}

Constituye una de las formas discursivas más complejas, no solo en su abordaje teórico, sino también en su dominio por parte de los estudiantes de nivel básico (Parodi, 2000, 2003), secundario (Concha \& Paratore, 2011) y universitario (Serrano de Moreno \& Villalobos, 2008).

Desde la perspectiva dialógica-racional, la argumentación puede ser definida como una: 
"actividad verbal y social cuya meta es reforzar o debilitar la aceptabilidad de un punto de vista controvertido ante un oyente o un lector, emitiendo una constelación de proposiciones destinadas a justificar (o refutar) ese punto de vista ante un juez racional" (van Eemeren, Grootendorst, Snoek Henkemans, Blair, Johnson, Krabbe, Plantin, Walton, Willard, Woods \& Zarefsk (1996) citado por Maingueneau \& Charaudeau, 2005: 46).

A lo anterior van Eemeren, Grootendorst y Snoeck Henkemans (2006) lo han denominado discusión crítica, es decir, la interacción entre un protagonista que defiende un punto de vista positivo o negativo frente a las objeciones de un antagonista que lo discute $y$, a su vez, defiende un punto de vista opuesto. La discusión crítica puede estar implícita cuando la argumentación asume la forma de un monólogo en el que solo se manifiesta la presencia del protagonista. Sin embargo, este monólogo puede ser visto como un diálogo ya que la argumentación siempre mantiene su objetivo de convencer a un opositor virtual o real.

Cuando la argumentación es directamente contraria a una postura preexistente, se utiliza la refutación, es decir, un "acto reactivo argumentativo de oposición" (Maingueneau \& Charaudeau, 2005: 491). Su propósito es destruir el discurso argumentativo del contrario.

\subsection{El hipertexto}

Entre los diversos recursos de lectura a los cuales pueden recurrir los escritores involucrados en tareas del tipo 'leer para escribir' se encuentra el hipertexto.

Podemos reconocer un sistema de hipertexto por cinco elementos básicos que lo componen (Lamarca, 2006): los documentos o archivos, que son conjuntos de información independiente que se presentan como una unidad; los nodos, o porciones de información (palabras, frases, imágenes, etc.) que entran en relación con otros nodos a los que proporcionan acceso; los enlaces, que son las conexiones entre segmentos de información; los anclajes de los nodos o el inicio y destino de cada enlace y los mapas de navegación que constituyen meta-nodos, con información sobre otros nodos y pueden estar configurados como índices, trayectos o sumarios.

Respecto de la estructura del sistema hipertextual -entendida como la forma en que se organiza la información y la interconexión de los nodos en la arquitectura de la web (Burin, et al., 2010)- Landow (2009) acepta la existencia de un hipertexto secuencial, el cual puede tener un soporte impreso. Consiste en un texto principal en el cual se intercalan números o símbolos que señalan las notas a pie de página o al final del texto. El lector puede dejar la lectura principal para seguir alguna de estas conexiones y continuar leyendo otras notas, volver al texto principal, y volver a dejarlo para seguir la siguiente nota al pie. La diferencia con el hipertexto electrónico es que éste facilita el 
seguimiento de conexiones que conducen a información que se encuentra físicamente lejos de sus referencias.

La estructura de hipertexto secuencial (llamada 'jerárquica' por Burin, Barreyro, Saux \& Irrazábal, 2015) consiste en un texto jerarquizado al que se le añade información complementaria a través de enlaces unidireccionales que no se conectan con otros enlaces, lo que mantiene un orden de lectura mayormente controlado por el autor del hipertexto. También pueden darse los casos de estructura jerárquica con un menú principal que contiene los enlaces a los nodos que conforman el cuerpo del texto (Burin et al., 2015).

Por otro lado, la estructura hipertextual en red está conformada por un entramado de enlaces que se interrelacionan de manera horizontal cuyo camino de lectura debe ser construido por el propio lector, debido a que no existe una entrada ni una salida única del hipertexto (Landow, 2009). Suele ser representada en forma de conglomerado: nube de tags o enlaces en la página donde están alojados (Burin et al., 2010).

El hipertexto es un recurso permanentemente utilizado por los estudiantes en el contexto educativo en todos sus niveles, lo cual tiene diversas implicancias. Por una parte, se debe considerar que los documentos digitales, por su naturaleza en red y su inmediatez, otorgan una mayor libertad a los lectores al momento de construir su camino de lectura. Por otro lado, esta flexibilidad conlleva una carga extra al propio proceso de lectura y comprensión: la toma de decisiones respecto de qué enlaces seguir y qué nodos explorar para lograr el doble objetivo de comprender para posteriormente escribir un texto propio.

\subsection{Escritura digital en el contexto educativo}

La idea de que los materiales del entorno de la tarea y la tecnología de escritura tienen incidencia en los procesos y en los productos escritos de los escolares ha motivado a los investigadores a abordar este escenario desde diferentes perspectivas. Los estudios de Henao y Ramírez (2006); Henao, Chaverra, Bolívar, Puerta y Villa (2006); Henao y Álvarez (1991, citado por Henao \& Ramírez, 2006) apuntan a que el uso de recursos digitales (herramientas hipermediales, páginas web como fuentes de información y procesadores de texto) inciden en la motivación, la calidad de los productos escritos y en la valoración de los estudiantes del proceso mismo como una forma de aprendizaje.

En una línea similar a la del presente estudio, Kirkpatrick y Klein (2016) presentan una de las primeras investigaciones centradas en las estrategias utilizadas por nueve estudiantes de enseñanza media, escritores hábiles, en una tarea de leer para escribir un ensayo argumentativo a partir de recursos digitales. Identificaron dos estrategias 
principales: la creación de documentos mediadores de planificación o escribir su texto directamente a partir de los recursos leídos. Dichas estrategias estarían dirigidas a maximizar las ventajas (por ejemplo, la facilidad para modificar un documento electrónico) y aminorar las restricciones (el exceso de información disponible en Internet) que implica el uso de recursos electrónicos.

\subsection{Registro del proceso de composición escrita}

El método de registro de la actividad de escritura en el teclado del computador (keystroke logging) consiste en la observación sincrónica de los procesos de traducción y transcripción con la finalidad de inferir los procesos mentales que se llevan a cabo. La ventaja de los métodos de keystroke logging es su aplicación en entornos contemporáneos de escritura que tienen lugar en contextos digitales donde los escritores tienen fácil acceso a una amplia variedad de recursos. Típicas actividades relacionadas con lo anterior son la búsqueda de información y la lectura de recursos en línea. Ambas son tareas de alta exigencia, por la cantidad de información disponible y porque los materiales son más complejos.

Zhang y Deane (2015) han usado este sistema para analizar la producción de ensayos por parte de estudiantes de enseñanza media. En su estudio, encontraron relaciones significativas entre dos factores del proceso de producción -fluidez de la escritura y la planificación-deliberación- y la calidad del texto final (evaluada por jueces humanos). Ambas investigaciones utilizaron el software de registro de la actividad en el teclado desarrollado por la Educational Testing Service (ETS).

\subsection{Criterios para la evaluación de los productos escritos}

Una forma eficiente de evaluar la producción de textos argumentativos desde una perspectiva cognitiva-textual es organizar la evaluación en torno a los niveles del discurso propuestos por van Dijk (1980, 1985, 1996, van Dijk \& Kintsch, 1978, 1983): nivel superestructural, macroestructural y microestructural.

La microestructura designa la estructura local de un discurso, es decir, la estructura de las oraciones y las relaciones de conexión y de coherencia entre ellas. Está formada por una red de proposiciones o 'ideas de un hecho’ (van Dijk, 1996) relacionadas entre sí. Las relaciones pueden ser de diverso tipo: causales, de finalidad, de correferencia, entre otras, y se expresan frecuentemente mediante conectivos en la superficie textual.

La macroestructura designa el contenido global de un discurso y determina la coherencia global del mismo. Está constituida por macroproposiciones que se derivan de la microestructura textual por medio de reglas de proyección denominadas macroestrategias textuales. También se derivan macroproposiciones del conocimiento previo del lector, mediante macroestrategias contextuales. Estas macroproposiciones configuran el tema o asunto del discurso. 
La superestructura refiere a la forma global de un discurso, que define su ordenación y las relaciones jerárquicas de cada uno de sus componentes. Se describe en términos de categorías y de reglas de formación.

Parodi (2000) desarrolló una pauta de evaluación de los productos escritos argumentativos en escolares chilenos de 8vo año básico en conformidad con estos tres niveles. La pauta de evaluación se enfocó principalmente en medir el grado de coherencia y cohesión de los textos producidos por los estudiantes (nivel micro y macroestructural) y en su adecuación al esquema básico de los textos argumentativos (nivel superestructural). A continuación, presentamos el detalle de los aspectos a evaluar según Parodi (2000), por cada nivel discursivo.

Nivel microestructural: correferencia y relaciones interoracionales de causa-efecto.

Nivel macroestructural: tema o tópico, adecuación a la situación comunicativa y un mínimo de dos macroproposiciones-argumento.

Nivel superestructural: presencia de tesis explícita, relacionada con la situación comunicativa, contener a lo menos un argumento con uno o más hechos que lo apoyen y la conclusión.

\begin{tabular}{lllll}
\hline Level & Scoring & & Total & \\
\hline Microstructural & & 3 & 5 & 5 \\
$\quad$ Nominal co-reference & 1 & 3 & 5 & 5 \\
Nominal ellipsis & 1 & 3 & 5 & 5 \\
Cause-effect relation & 1 & & & \\
\hline Macrostructural & & 5 & 9 & 9 \\
Macroproposition 1 & 1 & 5 & 9 & 9 \\
Macroproposition 2 & 1 & 5 & 9 & 9 \\
Topic & 1 & & & 9 \\
\hline Superstructural & & 5 & 9 & 9 \\
Thesis & 1 & 5 & 9 & \\
Arguments & 1 & 5 & & \\
$\quad$ Conclusion & 1 & & & \\
Total Test & 69 & & & \\
\hline
\end{tabular}

Figura 2. Pauta general de evaluación del texto argumentativo escrito (Parodi, 2007: 245). 


\section{Marco metodológico}

\subsection{Participantes}

El estudio se realizó en un establecimiento educacional de dependencia municipal de la comuna de Concepción, Región del Biobío.

La muestra inicial estuvo constituida por 131 estudiantes de cuarto año medio de ambos sexos, cuyas edades fluctuaban entre los 17 y los 19 años. Al consultarles por medio de una encuesta por sus habilidades previas de lectura y escritura en el computador, el $77,8 \%$ declaró poseer un grado alto o muy alto de experiencia navegando en internet con la finalidad de aprender sobre un tema; mientras que el 63,3\% afirmó que su experiencia escribiendo en el teclado del computador con la finalidad de producir un texto usando Microsoft Office Word era alta o muy alta. Cabe señalar que en tercer año medio los estudiantes habían aprendido a planificar, escribir y corregir textos ensayísticos durante la unidad alusiva al texto ensayístico perteneciente al programa de estudios del Ministerio de Educación de Chile.

De este grupo inicial finalmente fueron seleccionados 80 sujetos en base a su desempeño en una versión resumida de la prueba de comprensión lectora Lectum 7 (Riffo, Véliz, Castro, Reyes, Figueroa, Salazar \& Herrera, 2011)² que constaba de 18 preguntas, a cada una de las cuales se les asignó el valor de 1 punto.

El criterio de selección de los sujetos participantes consistió en recoger los puntajes extremos, es decir, a los cuarenta que obtuvieron los rendimientos más altos y a los cuarenta que obtuvieron los más bajos, descartando a 51 sujetos que obtuvieron puntajes intermedios. De esta forma, la muestra estuvo compuesta de 40 lectores con mayor habilidad lectora (entre 9 y 15 puntos en Lectum 7) y 40 con menor habilidad lectora (entre 1 y 6 puntos). Se eligió a los sujetos que obtuvieron los puntajes extremos con el objeto de encontrar diferencias significativas entre ambos grupos en su desempeño en la tarea según su nivel de habilidad lectora (hábil o menos hábil).

Esta muestra fue separada en dos subgrupos, de acuerdo al tipo de hipertexto (jerarquizado o en red) que les correspondería leer para posteriormente producir un texto argumentativo. Se procuró distribuir de forma equilibrada a los sujetos en cada subgrupo, de forma que cada uno tuviera una cantidad similar de puntajes superiores e inferiores dentro de su propio grupo. De los 80 sujetos seleccionados, por diferentes razones solo 69 realizaron la totalidad del experimento. Finalmente, se descartó a un sujeto cuya actividad experimental arrojó datos anormales, quedando la muestra final de 68 sujetos compuesta de la siguiente manera: 
Tabla 1. Descripción de la muestra final.

\begin{tabular}{|l|c|c|c|c|}
\hline \multicolumn{1}{|c|}{ Habilidad lectora } & \multicolumn{2}{c|}{ Mayor habilidad } & \multicolumn{2}{c|}{ Menor habilidad } \\
\hline \multicolumn{1}{|c|}{ Hipertexto } & Jerarquizado & En red & Jerarquizado & En red \\
\hline Promedios nota Lectum 7 & 4,1 & 4,1 & 2,3 & 2,5 \\
\hline D. E. & 0,59 & 0,69 & 0,4 & 0,18 \\
\hline Promedio memoria operativa & 4,3 & 4,7 & 4,2 & 4,2 \\
\hline D. E. & 0,88 & 1,14 & 0,85 & 1,12 \\
\hline$N^{\circ}$ de sujetos & 19 & 19 & 16 & 14 \\
\hline
\end{tabular}

\subsection{Diseño de investigación}

Corresponde a un diseño experimental factorial en el que se pretende dar cuenta de las posibles relaciones entre la habilidad lectora y la estructura un hipertexto -utilizado como recurso de lectura- en los procesos de escritura y en la calidad de los productos escritos argumentativos de estudiantes de enseñanza media, desde un punto de vista cuantitativo.

La variable independiente habilidad lectora fue medida a través de la aplicación de Lectum 7 (Riffo et al., 2011), en base a las calificaciones obtenidas por los sujetos. La variable independiente estructura del recurso hipertextual, en tanto, fue manipulada mediante la elaboración de dos hipertextos, uno jerarquizado y otro en red.

La variable dependiente proceso de producción escrita digital fue operacionalizada a través de la medición de los siguientes índices: pausas (cantidad y duración en segundos), revisiones (cantidad), visitas al recurso de lectura hipertextual (cantidad y duración en segundos). La variable dependiente calidad de los productos escritos fue medida a través de una pauta adaptada de la pauta de evaluación de la producción de textos escritos argumentativos de Parodi (2000). Dicho instrumento, validado por el autor en escolares de octavo año básico, fue modificado para adecuarlo a la exigencia correspondiente a estudiantes de cuarto año de enseñanza media.

\subsection{Materiales}

a) Encuesta de interés de lectura. Fue aplicada a la totalidad de la muestra inicial en la que se les inquiría por el grado de interés y el nivel de conocimiento previo sobre cuatro temas, esto con el fin de seleccionar el hipertexto que se utilizaría como recurso de lectura. En esta encuesta, además, se consultó a los sujetos sobre su experiencia en el manejo del computador (navegación en internet con la finalidad de aprender y uso del software Microsoft Word).

b) Lectum 7 (Riffo et al., 2011). Esta prueba mide la comprensión lectora en tres dimensiones: textual, pragmática y crítica. Abarca todos los niveles de escolaridad, desde Kinder (Lectum 1) hasta Tercer y Cuarto Año Medio (Lectum 7). Para este estudio se utilizó una versión resumida que constaba de dos textos: uno narrativo y otro ensayístico, que fueron seleccionados por su 
mayor cercanía con el hipertexto escogido por los mismos sujetos. Para Lectum se utilizaron tres diferentes métodos para el establecimiento de la confiabilidad $^{3}$, los cuales oscilaron entre 0.58 y 0.87 mostrando una confiabilidad, establecida mediante consistencia interna, aceptable y medianamente alta para una prueba de aprovechamiento o de habilidad.

c) InputLog (Leijten \& Van Waes, 2013). Durante el experimento, se recabaron datos correspondientes a los procesos de escritura gracias al software InputLog versión 7.0.0.11, que realiza un registro de los datos del proceso de escritura en teclado. Este registro arrojó un archivo .idfx por sujeto, el cual fue sometido a los siguientes análisis: pausas, revisiones y análisis general.

d) Recurso de lectura hipertextual. Un artículo de una revista de divulgación científica, cuyo tema fue el preferido por los estudiantes en la encuesta de interés de lectura, fue reorganizado según un esquema macroestructural deductivo en el que se basó el hipertexto de estructura jerarquizada. La página de inicio contenía enlaces a diez temas y subtemas organizados de lo general a lo particular, mientras que cada ventana enlazada contaba con una barra menú al costado izquierdo, encabezada por el enlace a la página de inicio y seguida por diez enlaces numerados correspondientes a cada tema y subtema del hipertexto. El bloque de texto de las ventanas no contenía enlaces. La página de inicio del hipertexto en red presentaba los mismos enlaces del hipertexto jerarquizado, pero dispuestos en forma de nube por orden alfabético según la letra inicial de cada tema o subtema. Cada ventana enlazada contenía un bloque de texto dentro del cual se incorporaron dos enlaces correspondientes a palabras o frases que tenían relación con el tema de la ventana a la cual conducían.
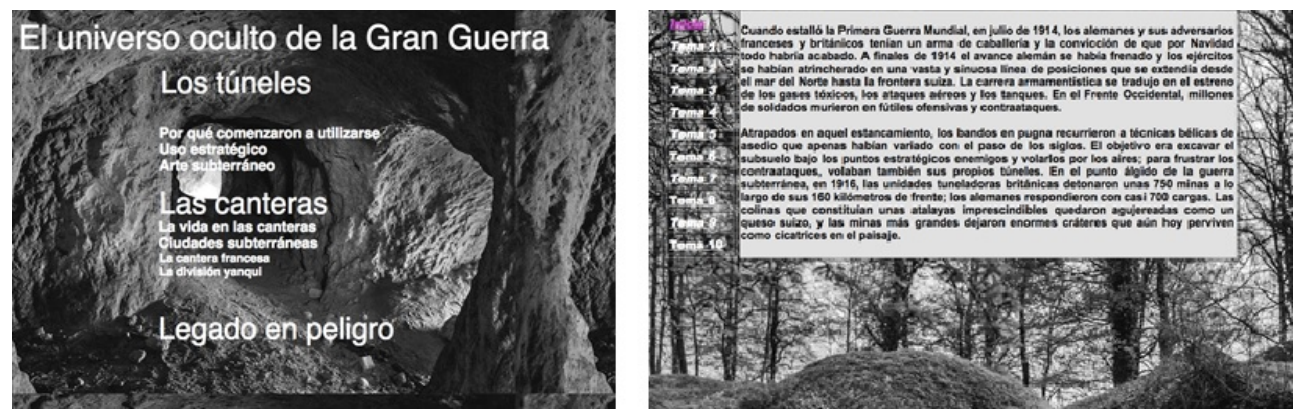

Figura 3. Página de inicio y ventana del hipertexto jerarquizado. Fuente: Elaboración propia. 

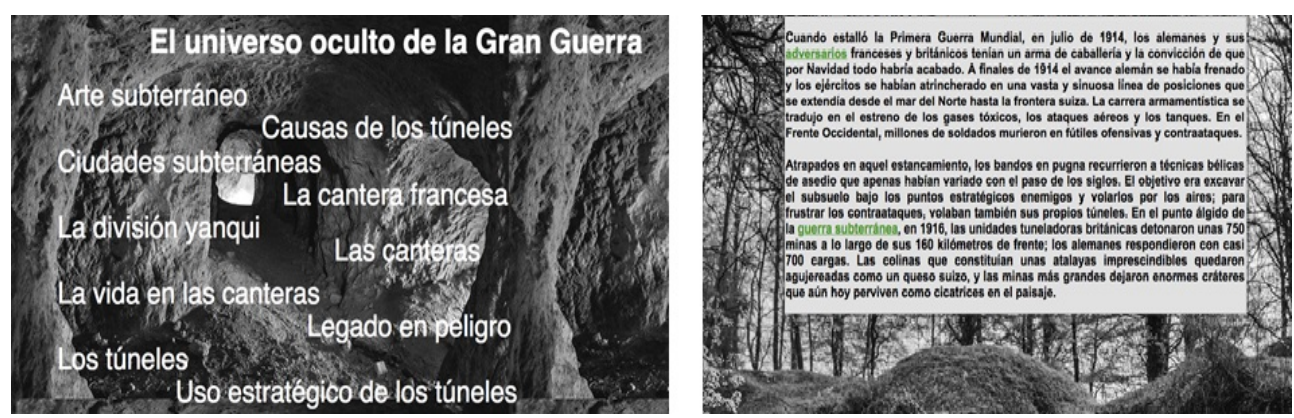

Figura 4. Página de inicio y ventana del hipertexto en red. Fuente: Elaboración propia.

e) Pauta de evaluación de textos escritos argumentativos. Se aplicó una adaptación de la pauta de evaluación de la producción de textos escritos argumentativos de Parodi (2000) validada mediante análisis estadístico con una confiabilidad de 0,9129 en su primera aplicación (Parodi, 2000), y con 0,82 según estimación de la fórmula KR20 (Parodi, 2007). Las modificaciones, que se realizaron en los niveles macro y superestructural, consistieron en incorporar una tercera macroproposición a las dos exigidas por la pauta original, y sustituir la superestructura original conformada por una tesis, argumentos y conclusión por una superestructura argumentativa dialéctica, es decir, compuesta de tesis, argumento, contraargumento, refutación y conclusión.

\subsection{Procedimiento}

Fase 1: Los sujetos acudieron a una primera sesión por grupos de veinte y fueron situados cada uno frente a un computador, intercalando entre ellos un espacio vacío para facilitar la concentración. Como instrucción, se les indicó que debían leer todos los contenidos de la página web 'El universo oculto de la Primera Guerra Mundial', navegando por todas las ventanas, tratando de comprender lo mejor posible la información, ya que después de leer tendrían que responder preguntas de comprensión. No podían ingresar a otras páginas web una vez iniciada la lectura, ni utilizar sus teléfonos celulares. A los sujetos que debían leer el hipertexto jerarquizado se les solicitó ingresar a la página web www.granguerra-uno.com, mientras que a los sujetos que debían leer la versión en red se les solicitó ingresar a www.granguerrados.com.

Fase 2:

1. Test de velocidad de escritura en el teclado: se le solicitó a una submuestra de 47 sujetos rendir un pretest que consistió en escribir su nombre completo sin errores diez veces en líneas consecutivas. El promedio de duración de las pausas ejecutadas durante esta tarea de escritura altamente automatizada permitió fijar el umbral de pausas con implicancia cognitiva en 0,274 segundos. 
2. Actividad experimental: Los sujetos debían producir un ensayo argumentativo breve basándose en la misma página web leída durante la sesión anterior en una hoja de procesador de texto. En primer lugar, se les detalló el manejo de InputLog, explicando que todo el proceso de escritura en el teclado del computador sería registrado mediante este programa informático. Luego de ejecutar el programa, completaron sus datos (nombre y edad) en la pestaña "Session identification" y seleccionaron la opción "New document". A continuación, los sujetos escribieron su ensayo en la hoja de Word que se abrió automáticamente al presionar el botón de grabación del programa InputLog. Mientras escribían, podían visitar la página web "El universo oculto de la Gran Guerra", si lo consideraban necesario. A cada sujeto se le entregó un papel donde estaba escrita la dirección de la página web que le correspondió leer durante la sesión anterior.

Las instrucciones, que fueron entregadas por escrito y leídas en voz alta, fueron las siguientes: a partir de la pregunta ‘¿Deberían enseñarse en la clase de Historia los hechos que se exponen en el texto El universo oculto de la Gran Guerra?', producir un ensayo breve compuesto de cinco partes, cada una en un párrafo (introducción argumento - contraargumento - refutación - conclusión); de considerarlo necesario, releer el hipertexto "El universo oculto de la Gran Guerra" para apoyar la argumentación. Al finalizar la sesión, los archivos que contenían la información de la actividad experimental de cada sujeto fueron recuperados desde sus respectivos computadores para su posterior análisis.

\subsection{Proceso de recolección de datos}

1. Sondeo inicial para seleccionar el hipertexto: la recolección de datos se inició con una encuesta en la que se inquiría por el grado de interés y el nivel de conocimiento previo sobre cuatro temas. Además, se consultó a los sujetos sobre su experiencia en el manejo del computador (navegación en internet con la finalidad de aprender y uso del software Microsoft Word).

2. Aplicación de Lectum 7: a esta misma muestra se le administró una versión resumida de la prueba Lectum 7 de comprensión lectora (Riffo et al., 2011), consistente en dos textos: uno narrativo y otro ensayístico, que fueron seleccionados por su mayor cercanía con el hipertexto escogido por los mismos sujetos. Esta prueba se utilizó para seleccionar a los sujetos que obtuvieron puntajes extremos. Los puntajes intermedios fueron eliminados.

3. Test de Memoria Operativa: a esta nueva muestra reducida se le administró una prueba para medir su nivel de memoria operativa, el Test de Dígitos Inversos (Wechsler, 1995). En sesiones individuales, se solicitó a los sujetos escuchar y repetir en voz alta, respetando el orden dado, diferentes series de números que iban aumentando en longitud a medida que los sujetos lograban repetir correctamente cada 
serie. A cada sujeto se le asignó un punto por cada serie recordada en el orden correcto, con un máximo de 8 puntos.

4. Registro del proceso de escritura digital con InputLog: durante el experimento, se recabaron datos correspondientes a los procesos de escritura gracias al software InputLog versión 7.0.0.11, que realiza un registro de los datos del proceso de escritura en teclado. Este registro arrojó un archivo .idfx por sujeto, el cual fue sometido a los siguientes análisis: pausas, revisiones y análisis general. Este último se utilizó para determinar la cantidad de ocasiones en que los sujetos tuvieron como foco principal en la pantalla del computador, el recurso de lectura desde la tarea principal, consistente en la producción de un texto argumentativo. Estos análisis arrojaron sendos archivos .XML

Tabla 2. Índices recabados por cada tipo de análisis efectuado con InputLog.

\begin{tabular}{|c|l|l|l|}
\hline \multicolumn{1}{c|}{$\begin{array}{c}\text { Análisis } \\
\text { InputLog }\end{array}$} & \multicolumn{1}{|c|}{$\begin{array}{c}\text { Análisis de } \\
\text { pausas }\end{array}$} & \multicolumn{1}{c|}{$\begin{array}{c}\text { Matriz de } \\
\text { revisiones }\end{array}$} & \multicolumn{1}{c|}{ Análisis general } \\
\hline \multirow{2}{*}{$\begin{array}{l}\text { Índices } \\
\text { recabados }\end{array}$} & $\begin{array}{l}\text { Número de } \\
\text { pausas }\end{array}$ & $\begin{array}{l}\text { Número de } \\
\text { revisiones }\end{array}$ & $\begin{array}{l}\text { Número de visitas al recurso de lectura } \\
\text { hipertextual desde el documento principal }\end{array}$ \\
\cline { 2 - 4 } & $\begin{array}{l}\text { Duras (s) } \\
\text { pausas }\end{array}$ & Duración de las visitas (s) \\
\hline
\end{tabular}

Al mismo tiempo que se recuperaron los archivos del proceso de escritura, se recogieron los productos escritos de los sujetos en formato de archivo Word (.doc). Para evaluar los productos escritos se aplicó la adaptación de la pauta de evaluación de la producción de textos escritos argumentativos de Parodi, ya mencionada. La pauta fue aplicada por una jueza experta, con cinco años de experiencia como docente en el área de lenguaje ${ }^{4}$. La corrección se realizó directamente sobre los archivos digitales y se incorporaron comentarios sobre los trabajos de los sujetos para justificar el puntaje asignado en cada ítem.

\section{Resultados}

La base de datos fue analizada con el software SPSS 24.0. Las variables numéricas fueron representadas por sus medidas de tendencia central y de dispersión (media, desviación estándar y cuartiles). En los índices de comprensión y de producción textual se analizó el puntaje total por cada dimensión (microestructural, macroestructural y superestructural), así como también por cada uno de sus subcomponentes. Se utilizó el test t de Student (U de Mann-Whitney) para comparar dos variables (índices del proceso de escritura y la producción textual) entre los dos grupos de hipertexto. Se obtuvo el coeficiente de correlación de Spearman para estudiar la relación entre la variable nivel de comprensión lectora (rendimiento en Lectum 7) y la variable producción textual (índices del proceso de escritura y la calidad de los productos escritos). Se utilizó un nivel de significación de 0,05. 


\subsection{Resultados del proceso de escritura}

A continuación, se describen los resultados registrados con InputLog en cada uno de los cinco índices del proceso de escritura. La tabla 2 confronta los índices obtenidos por sujetos que leyeron un hipertexto jerarquizado con los que leyeron un hipertexto en red. No se observaron diferencias significativas entre ambos grupos en ninguno de los cinco índices. Por su parte, la Tabla 3, que expone las correlaciones entre el rendimiento en Lectum 7 y los índices del proceso de escritura, tampoco arroja correlaciones significativas.

Tabla 3. Cuantificación de los índices del proceso de escritura y comparación por tipo estructura hipertextual.

\begin{tabular}{|c|c|c|c|c|c|c|c|c|c|c|c|c|c|c|}
\hline \multirow{2}{*}{\multicolumn{2}{|c|}{ Variable }} & \multicolumn{5}{|c|}{ Jerarquizado $(\mathrm{n}=35)$} & \multicolumn{5}{|c|}{ En red $(n=33)$} & \multirow{2}{*}{ Estadígrafo } & \multirow{2}{*}{ valor $\mathrm{p}$} & \multirow[b]{2}{*}{$\eta^{2}$} \\
\hline & & Media & D.E. & Mediana & Q1 & Q3 & Media & D.E. & Mediana & Q1 & Q3 & & & \\
\hline $\mathrm{u}$ & Número de revis & 192,9 & 182,2 & 1650 & 79,0 & 218,0 & 213,3 & 133,4 & & 125,0 & 268,0 & 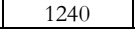 & 2129 & 0,0041 \\
\hline 0 & Núm & 1026,1 & 339,6 & & 802,0 & 1239,0 & 1034,7 & 368,0 & & 812,0 & 1323,0 & & & 02 \\
\hline $\mathrm{T}$ & Durac & 1,5 & 0,4 & & 1,2 & 1,8 & 1,4 & 0,3 & 1, & 1,2 & 1,7 & 88 & 21 & 0,0116 \\
\hline $\mathrm{U}$ & Número de visitas & 5,9 & 4,6 & 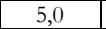 & 2,0 & 8,0 & 7,4 & 5,2 & 0,0 & 3,0 & 11,0 & 1243 & 0,1979 & 0,0247 \\
\hline 1 & Duración visitas & 434,9 & 310,4 & 423,0 & 175,0 & 651,7 & 346,4 & 287,7 & 264,1 & 111,7 & 575,4 & 1043 & 0,2388 & 0,0219 \\
\hline
\end{tabular}

Tabla 4. Correlaciones entre el rendimiento en Lectum 7 y los índices del proceso de escritura.

\begin{tabular}{|c|c|c|c|c|c|c|c|}
\hline & & 1. & 2. & 3. & 4. & 5. & 6. \\
\hline \multirow{2}{*}{ 1. LECTUM 7} & Coeficiente de correlación & 1 & 0,017 & 0,015 & $-0,003$ & 0,068 & 0,167 \\
\hline & Sig. (bilateral) & t & 0,888 & 0,904 & 0,98 & 0,579 & 0,173 \\
\hline \multirow{2}{*}{ 2. Número de revisiones } & Coeficiente de correlación & & 1 & , 375** & $-0,214$ & $-0,107$ & $-0,176$ \\
\hline & Sig. (bilateral) & & . & 0,002 & 0,08 & 0,385 & 0,152 \\
\hline \multirow{2}{*}{ 3. Número de pausas } & Coeficiente de correlación & & & 1 &,$- 345^{* *}$ & $-0,059$ & $-0,134$ \\
\hline & Sig. (bilateral) & & & & 0,004 & 0,633 & 0,276 \\
\hline \multirow{2}{*}{ 4. Duración de pausas } & Coeficiente de correlación & & & & 1 & 0,035 &, $530 * *$ \\
\hline & Sig. (bilateral) & & & & . & 0,775 & 0 \\
\hline \multirow{2}{*}{ 5. Número de visitas } & Coeficiente de correlación & & & & & 1 & 0,228 \\
\hline & Sig. (bilateral) & & & & & $\cdot$ & 0,062 \\
\hline \multirow{2}{*}{ 6. Duración de las visitas } & Coeficiente de correlación & & & & & & 1 \\
\hline & Sig. (bilateral) & & & & & & \\
\hline
\end{tabular}

**. La correlación es significativa al nivel 0,01 (bilateral).

\subsection{Resultados de los productos escritos}

A continuación, se presenta una descripción de los resultados obtenidos por los sujetos en la evaluación de sus ensayos argumentativos y las correlaciones encontradas con su habilidad lectora -determinada por su rendimiento en la Prueba Lectum 7- y las diferencias según la estructura del hipertexto leído -jerarquizada o en red.

La Tabla 4 muestra que existe una correlación positiva entre la habilidad lectora y la calidad de los textos producidos por los sujetos, en los tres niveles discursivos medidos por la pauta de evaluación. En otras palabras, los sujetos que lograron puntajes superiores en Lectum 7, fueron evaluados con mayores puntuaciones en sus ensayos en los niveles microestructural, macroestructural y superestructural. Tal como 
indica la tabla, la correlación con la habilidad lectora es más significativa cuando se focaliza en la calidad general de los textos, es decir, en los puntajes totales obtenidos por los ensayos.

Tabla 5. Correlaciones entre el rendimiento en Lectum 7 y la calidad de los ensayos.

\begin{tabular}{|c|c|c|c|c|c|c|}
\hline & & 1. & 2. & 3. & 4. & 5. \\
\hline \multirow{2}{*}{ 1. LECTUM 7} & Coeficiente de correlación & 1 & ,284* &, $302^{*}$ & ,295* & $347^{* *}$ \\
\hline & Sig. (bilateral) & . & 0,019 & 0,012 & 0,015 & 0,004 \\
\hline \multirow{2}{*}{ 2. Microestructura } & Coeficiente de correlación & & 1 & 0,18 & 0,115 & $327^{* *}$ \\
\hline & Sig. (bilateral) & & $\cdot$ & 0,142 & 0,349 & 0,006 \\
\hline \multirow{2}{*}{ 3. Macroestructura } & Coeficiente de correlación & & & 1 &, $874^{* *}$ & ,918** \\
\hline & Sig. (bilateral) & & & . & 0 & 0 \\
\hline \multirow{2}{*}{ 4. Superestructura } & Coeficiente de correlación & & & & 1 &, $936^{* *}$ \\
\hline & Sig. (bilateral) & & & & . & 0 \\
\hline \multirow{2}{*}{ 5. Calidad general del texto } & Coeficiente de correlación & & & & & 1 \\
\hline & Sig. (bilateral) & & & & & . \\
\hline
\end{tabular}

*. La correlación es significativa al nivel 0,05 (bilateral).

**. La correlación es significativa al nivel 0,01 (bilateral).

Sin embargo, tal como muestra la Tabla 5, si comparamos los puntajes obtenidos por los ensayos de los sujetos que leyeron un hipertexto jerarquizado con aquellos que leyeron un hipertexto en red, no es posible encontrar diferencias significativas entre los grupos en ninguno de los niveles evaluados en la pauta, así como tampoco al comparar los puntajes globales de los ensayos.

Tabla 6. Comparación de la calidad de los ensayos por estructura del hipertexto.

\begin{tabular}{|c|c|c|c|c|c|c|c|c|c|c|c|c|c|c|}
\hline & \multirow{2}{*}{ Variable } & \multicolumn{5}{|c|}{ Jerarquizado $(n=35)$} & \multicolumn{5}{|c|}{ En red $(n=33)$} & \multirow{2}{*}{ Estadígrafo } & \multirow{2}{*}{ valor $\mathrm{p}$} & \multirow{2}{*}{$\eta^{2}$} \\
\hline & & Media & D.E. & Mediana & Q1 & Q3 & Media & D.E. & Mediana & Q1 & Q3 & & & \\
\hline $\mathrm{u}$ & Micro & 13,63 & 1,73 & 15 & 13 & 15 & 13,36 & 1,9 & 13 & 13 & 15 & $-0,61$ & 0,5436 & 0,07 \\
\hline $\mathrm{u}$ & Macro & 22,43 & 6,87 & 27 & 23 & 27 & 21,42 & 7,14 & 23 & 19 & 27 & $-0,86$ & 0,3914 & 0,1 \\
\hline $\mathrm{u}$ & Super & 40,43 & 5,99 & 41 & 41 & 45 & 38,82 & 7,15 & 41 & 37 & 45 & $-0,91$ & 0,3638 & 0,11 \\
\hline u & Total & 76,49 & 12,7 & 83 & 73 & 85 & 73,61 & 14,4 & 79 & 67 & 85 & $\begin{array}{l}-0,69 \\
\end{array}$ & 0,4889 & 0,08 \\
\hline
\end{tabular}

\subsection{Análisis comparativo entre las habilidades de comprensión y producción textual}

La Tabla 6 presenta las correlaciones entre las habilidades de comprensión y producción textual demostradas por los sujetos en Lectum 7 y en los productos escritos argumentativos, respectivamente. Se observan correlaciones positivas y estadísticamente significativas, aunque bajas, entre ambas habilidades en los niveles microestructural y macroestructural. En otras palabras, los sujetos que respondieron correctamente a las preguntas de comprensión microestructural y macroestructural de la Prueba Lectum 7 produjeron ensayos de mejor calidad en estos niveles textuales. En el nivel superestructural, en tanto, no se observaron diferencias significativas entre los grupos ni en comprensión ni en producción. 
Tabla 7. Correlaciones entre comprensión lectora y producción textual por nivel discursivo-textual.

\begin{tabular}{|c|c|c|c|}
\hline Comprensión & Producción & Spearman & p-valor \\
\hline Microestructura LECTUM & Microestructura & 0,28 & 0,0232 \\
\hline Macroestructura LECTUM & Macroestructura & 0,29 & 0,0157 \\
\hline
\end{tabular}

\section{Discusión}

Los resultados obtenidos no nos permiten relacionar la variable estructura del hipertexto con los índices del proceso de escritura ni con la calidad de los productos escritos, debido a que no se evidenciaron diferencias significativas entre ambos grupos de hipertexto. Por su parte, la variable habilidad lectora tampoco demostró tener relación con los índices del proceso de escritura. Sin embargo, sí es posible relacionar la habilidad lectora con la calidad de la escritura, ya que se observó una correlación positiva entre la habilidad lectora medida por el desempeño en Lectum 7 y la calidad global de los textos producidos por los sujetos, así como también con cada uno de los niveles (microestructural, macroestructural y superestructural) medidos por la pauta de evaluación.

Parodi (2007) logró establecer correlaciones significativas entre lectura y escritura en los tres niveles discursivo-textuales: la correlación fue moderada en el nivel microestructural $(0,57)$ y buena en los niveles macroestructural y superestructural $(0,68$ y 0,79 respectivamente). Aunque los resultados obtenidos por este estudio siguen la línea de Parodi (2007), las correlaciones sólo son significativas en los niveles microestructural y macroestructural (0,28 y 0,29, respectivamente) y son bajas. Mientras que en el estudio de Parodi (2007) las conexiones entre lectura y escritura son más fuertes en el nivel superestructural, en el presente estudio es a la inversa, ya que la correlación es negativa y no significativa. Esto podría ser explicado considerando que el rendimiento en producción de la superestructura textual se comparó con el rendimiento en comprensión en una única pregunta que apuntaba a este nivel discursivo-textual. Tal vez habría sido posible evidenciar una correlación positiva entre comprensión y producción en el nivel superestructural si la cantidad de preguntas de comprensión hubiese sido mayor.

La principal diferencia con los hallazgos de Parodi (2007) fue el nivel de logro en la comprensión y producción de la superestructura textual. Mientras que en su estudio los sujetos demostraron menor habilidad en la comprensión y producción de la superestructura textual, en el presente estudio el bajo rendimiento sólo se dio en la habilidad de comprensión, ya que en la producción textual entre el 60 y 80\% de los sujetos lograron las puntuaciones máximas en la formulación de una superestructura argumentativa dialéctica. Aquí la explicación podría provenir del factor nivel de escolaridad, debido a que el estudio de Parodi (2007) comprendía a una muestra de 
sujetos de octavo año básico, nivel en el cual el discurso argumentativo no formaba parte del currículum nacional, al menos en el año en que se desarrolló el estudio. Los sujetos del presente estudio, en tanto, estaban cursando el cuarto año medio, nivel en que se les refuerza la habilidad argumentativa ya desarrollada en tercer año medio, por lo tanto, no es de extrañar que hayan demostrado mayor dominio de esta superestructura textual. Otro factor a considerar es que más de la mitad de los sujetos declararon poseer una experiencia alta o muy alta en actividades de escritura en entornos electrónicos, lo cual podría haber potenciado sus habilidades en la escritura argumentativa al construir un entorno de la tarea diferente de la escritura en papel, con estrategias que son posibles solo en el medio digital (Kirkpatrick \& Klein, 2016).

Asimismo, no fue posible demostrar la existencia de relaciones entre los índices del proceso de escritura y la calidad de los productos finales, lo que sí fue logrado por Zhang y Deane (2015) y Chan (2017). Esta diferencia puede ser explicada sobre la base de que los indicadores fueron diferentes en ambos estudios. Zhang y Deane (2015) midieron patrones de pausas/ráfagas de escritura con el fin de establecer el nivel de fluidez de los escritores, además de medir todas aquellas pausas que, por estar situadas en lugares estratégicos (inicio de palabras, interoracionales), podrían ser indicadoras de subprocesos como la planificación. En el presente estudio, en tanto, las pausas fueron medidas sobre un umbral preestablecido solo en cuanto a su cantidad y duración, sin considerar patrones de pausas ni su localización, ya que la finalidad no era localizar subprocesos específicos, sino cuantificar las que fueran indicadoras de mayor esfuerzo cognitivo, con el fin de encontrar diferencias en relación con las variables independientes en estudio.

Chan (2017) analizó los procesos y productos de la escritura argumentativa en el contexto de una tarea de leer para escribir en dos estudiantes posgraduados de L2. Sin embargo, se considera que, por lo reducido de la muestra, no es posible generalizar sus resultados, aunque, por otra parte, éstos estén en línea con los obtenidos por Zhang y Deane (2015), respecto de la relación entre fluidez en la escritura y la calidad del producto final.

Con respecto a las correlaciones positivas, pero bajas, encontradas entre la habilidad lectora y los productos escritos, una posible explicación podría ser las escasas diferencias encontradas entre los puntajes superiores e inferiores en Lectum 7. Como se pudo apreciar en la presentación de los resultados generales en esta prueba, el rendimiento general fue bajo, por lo cual no fue posible contar con grupos cuyo nivel de habilidad lectora estuviera marcadamente diferenciada.

\section{CONCLUSIONES}

Podemos afirmar que la habilidad lectora incide en la calidad de los ensayos argumentativos digitales producidos por sujetos que están en la etapa terminal de su 
educación media. Sin embargo, no es posible concluir que esta misma habilidad determine los procesos de escritura digital considerados en el estudio: pausas, revisiones y visitas al hipertexto. Cada sujeto parece utilizar estrategias de escritura digital que no se reflejan en los índices escogidos para el análisis, independientemente de su nivel de habilidad lectora. A futuro, se recomienda que las investigaciones se focalicen en uno o dos índices solamente y se registren patrones de ocurrencia, localización y fluidez que permitan un análisis más fino, en la línea de lo desarrollado por investigaciones anteriores utilizando InputLog.

La estructura del recurso hipertextual utilizado para estimular la escritura, por su parte, no parece tener un rol determinante ni en los procesos de escritura ni en la calidad final de los ensayos producidos. Tenemos presente que el modelo de producción de textos escritos de Hayes (2012) establece que la lectura es uno de los recursos necesarios para la producción, así como también los materiales de la tarea (en este caso, los hipertextos). Sin embargo, en vista de los resultados obtenidos en el ámbito de la producción de textos, la estructura del recurso de lectura hipertextual no tendría una influencia significativa sobre el proceso de transcripción descrito por Hayes.

Los resultados obtenidos en la presente investigación contribuyen fuertemente a la idea base del enfoque 'leer para escribir', en el sentido de que estas habilidades deben trabajarse en conjunto en todos los niveles de escolaridad. Así lo exigen las producciones textuales propias de los ámbitos de la educación superior y laboral. Aquí adquiere relevancia la tipología textual que se utiliza para motivar el uso efectivo de recursos de lectura, en vista del peligro que representa el copiar y pegar, especialmente en el ámbito de la escritura digital. El texto argumentativo dialéctico, por su gran complejidad, resulta ideal para desarrollar este tipo de tareas, ya que exige al escritor defender un punto de vista propio, además de confrontar de manera exitosa la postura de un supuesto opositor, frente a lo cual es muy difícil hacer un uso mecanizado de los recursos de lectura, sean éstos hipertextuales o no.

\section{REFERENCIAS BIBLIOGRÁFICAS}

Alamargot, D., Caporossi, G., Chesnet, D. \& Ros, C. (2011). What makes a skilled writer? Working memory and audience awareness during text composition. Learning and Individual Differences, 21(5), 505-516.

Burin, D., Kahan, E., Irrazabal, N. \& Saux, G. (2010). Procesos cognitivos en la comprensión de hipertexto: Papel de la estructura del hipertexto, de la memoria de trabajo, y del conocimiento previo. Ponencia presentada en el Congreso Iberoamericano de Educación Metas 2021, Buenos Aires, Argentina. 
Burin, D. I., Barreyro, J. P., Saux, G. \& Irrazábal, N. (2015). Navegación y comprensión de textos digitales: Estructuras de hipertexto, conocimientos previos del dominio y capacidad de memoria de trabajo. Electronic Journal of Research in Educational Psychology, 13(37), 529-550.

Cassany, D. \& Ayala, G. (2008). Nativos e inmigrantes digitales en la escuela. Participación educativa: revista del Consejo Escolar del Estado, 9(4), 57-75.

Chan, S. (2017). Using keystroke logging to understand writers' processes on a reading-into-writing test. Language Testing in Asia, 7(1), 1-27.

Concha, S. \& Paratore, J. R. (2011). Local coherence in persuasive writing: An exploration of Chilean students' metalinguistic knowledge, writing process, and writing products. Written Communication, 28(1), 34-69.

Delaney, Y. A. (2008). Investigating the reading-to-write construct. Journal of English for Academic Purposes, 7(3), 140-150.

Flower, L. (1987). The role of task representation in reading to write. Berkeley, CA: Center for the Study of Writing.

Flower, L. \& Hayes, J. R. (1981). A cognitive process theory of writing. College composition and communication, 32(4), 365-387.

Hayes, J. (1996). Un nuevo marco para la comprensión de lo cognitivo y lo emocional en la escritura. The science of writing, 1, 72 .

Hayes, J. (2012). Modeling and remodeling writing. Written communication, 29(3), 369388.

Henao, O. \& Ramírez S. D. (2006). Impacto de una experiencia de producción textual mediada por tecnologías de información y comunicación en las nociones sobre el valor epistémico de la escritura. Revista Educación y Pedagogía, 18(46), 223-238.

Henao, O., Chaverra, D., Bolívar B. W., Puerta, D. L. \& Villa, N. H. (2006). La producción escrita mediada por herramientas informáticas. La calidad textual, el nivel de aprendizaje y la motivación. Lectura y Vida, 27(2) 6-13.

Kirkpatrick, L. C. \& Klein, P. D. (2016). High-achieving High School students' strategies for writing from Internet-based sources of information. Journal of Writing Research, 8(1).

Lamarca, M. (2006). Hipertexto: El nuevo concepto de documento en la cultura de la imagen. Tesis Doctoral, Universidad Complutense de Madrid [en línea]. Disponible en: http://www.hipertexto.info 
Landow, G. (2009). Hipertexto 3.0: La teoría crítica y los nuevos medios en una época de globalización. Barcelona: Paidós.

Leijten, M. \& Van Waes, L. (2013). Keystroke logging in writing research: Using Inputlog to analyze and visualize writing processes. Written Communication, 30(3), 358-392.

Maingueneau, D. \& Charaudeau, P. (2005). Diccionario de análisis del discurso. Buenos Aires: Amarrortu Editores.

O'Hara, K. P., Taylor, A., Newman, W. \& Sellen, A. J. (2002). Understanding the materiality of writing from multiple sources. International journal of humancomputer studies, 56(3), 269-305.

Parodi, G. (2000). La evaluación de la producción de textos escritos argumentativos: Una alternativa cognitivo/discursiva. Revista Signos. Estudios de Lingüistica, 33(47), 151-166.

Parodi, G. (2003). Relaciones entre lectura y escritura: Una perspectiva cognitiva discursiva. Valparaíso: Editorial Universitaria de Valparaíso.

Parodi, G. (2007). Reading-writing connections: Discourse-oriented research. Reading and writing, 20(3), 225-250.

Riffo, B., Véliz, M., Castro, G., Reyes, F., Figueroa, B., Salazar, O. \& Herrera, M. O. (2011). LECTUM. Prueba de comprensión lectora. Proyecto Fondef D08i1179, Conicyt, Chile.

Serrano de Moreno, S. \& Villalobos, J. (2008). Niveles discursivos del texto argumentativo escrito por estudiantes de formación docente. Anuario del Doctorado en Educación: Pensar la Educación, 3, 151-188.

Stein, V. (1989). Exploring the cognition of reading-to-write (Reading-to-Write Report No. 5). Technical Report No. 24 [en línea]. Disponible en: https:// files.eric.ed.gov/fulltext/ED306595.pdf

Van Dijk, T. A. (1980). Texto y contexto. Cátedra, Madrid.

Van Dijk, T. A. (1985). Semantic discourse analysis. Handbook of discourse analysis, 2, 103-136.

Van Dijk, T. A. (1996). Estructura y funciones del discurso, una introducción interdisciplinaria a la lingüistica del texto ya los estudios del discurso. México D. F.: Siglo Veintiuno Editores.

Van Dijk, T. A. \& Kintsch, W. (1978). Toward a model of text comprehension and production. Psychological review, 85(5), 363. 
Van Dijk, T. A. \& Kintsch, W. (1983). Strategies of discourse comprehension. Nueva York: Academic Press.

Van Eemeren, F., Grootendorst, R. \& Snoeck Henkemans, F. (2006). Argumentación: Análisis, evaluación, presentación. Buenos Aires: Biblos.

Wechsler, D. (1995). Test de inteligencia para adultos (WAIS): Manual. Buenos Aires: Paidós.

Zhang, M. \& Deane, P. (2015). Process features in writing: Internal structure and incremental value over product features. ETS Research Report Series, 2015(2), 1 12.

\section{NOTAS}

${ }^{1}$ Este estudio fue realizado en coordinación con la candidata al grado de Magíster Camila González Bello, quien, en su tesis de magíster titulada "Lectura digital: navegación y comprensión lectora en dos estructuras de hipertexto", en una primera fase, analizó el comportamiento de los sujetos del estudio (lectores hábiles y menos hábiles) en la lectura y comprensión de dos estructuras de hipertexto, jerarquizado y en red. Fueron medidos los siguientes índices de navegación: tiempo de lectura, número de enlaces visitados, número de retornos a la página de inicio, número de enlaces en redes visitados y número de enlaces revisitados. También fueron recabados datos sobre el nivel de comprensión alcanzado tras la lectura del hipertexto.

${ }^{2}$ Esta prueba mide la comprensión lectora en tres dimensiones: textual, pragmática y crítica. Abarca todos los niveles de escolaridad, desde jardín infantil hasta cuarto año medio. Para este estudio se utilizó Lectum 7, que mide las habilidades de comprensión en estudiantes de cuarto año medio.

${ }^{3}$ El método de división por mitades con corrección de Spearman Brown, el coeficiente de confiabilidad Alfa de Cronbach $(\alpha)$ y el coeficiente de confiabilidad para pruebas paralelas.

${ }^{4}$ Originalmente, eran tres juezas expertas, con más de cinco años de experiencia y con estudios de postgrado, quienes evaluaron los textos. Sin embargo, debido a que no se logró un nivel de acuerdo estadísticamente aceptable entre las puntuaciones otorgadas por ellas, se seleccionó solo a la que demostró mejor aplicación de la pauta mediante la justificación del puntaje asignado a cada ensayo. 